\title{
Finding a Shortest Non-Zero Path in Group-Labeled Graphs via Permanent Computation*
}

\author{
Yusuke Kobayashi $^{\dagger} \quad$ Sho Toyooka ${ }^{\ddagger}$
}

December 14, 2015

\begin{abstract}
A group-labeled graph is a directed graph with each arc labeled by a group element, and the label of a path is defined as the sum of the labels of the traversed arcs. In this paper, we propose a polynomial time randomized algorithm for the problem of finding a shortest $s-t$ path with a non-zero label in a given group-labeled graph (which we call the Shortest Non-Zero Path Problem). This problem generalizes the problem of finding a shortest path with an odd number of edges, which is known to be solvable in polynomial time by using matching algorithms. Our algorithm for the Shortest Non-Zero Path Problem is based on the ideas of Björklund and Husfeldt (2014). We reduce the problem to the computation of the permanent of a polynomial matrix modulo two. Furthermore, by devising an algorithm for computing the permanent of a polynomial matrix modulo $2^{r}$ for any fixed integer $r$, we extend our result to the problem of packing internally-disjoint $s$ - $t$ paths.
\end{abstract}

\section{Introduction}

The shortest path problem is one of the most well-studied problems in combinatorial optimization. In the problem, the objective is to find a shortest path connecting two specified vertices $s$ and $t$ in a given graph, and it can be done easily by the breadth first search if each edge has a unit length. For the shortest path problem in undirected (or directed) graphs with non-negative edge lengths, many polynomial time algorithms are proposed, such as Dijkstra's algorithm [3] and the Bellman-Ford algorithm [1]. As an extension of the shortest path problem, we can consider the problem with a parity constraint: given an undirected graph $G=(V, E)$, two specified vertices $s$ and $t$, and a non-negative length of each edge, find a shortest odd (or even) $s$ - $t$ path. Here, a path is said to be odd (resp. even) if it contains odd (resp. even) number of edges. Actually, this problem can be reduced to the weighted matching problem (see e.g. [12, Section 29.11e] and [7]), and hence it can be solved in polynomial time with the aid of weighted matching algorithms. Note that the directed variant is much harder than the undirected case, namely, it is NP-hard to test whether a given directed graph contains an odd (or even) directed path from $s$ to $t[9]$. We also note that we can easily find a shortest odd (or even) $s$-t walk in a given (directed) graph by standard dynamic programming.

As a generalization of the parity constraints, group-labeled graphs have been investigated [8], where a group-labeled graph is a directed graph with each arc labeled by a group element. In a group-labeled graph, the label of a path is defined as the sum of the labels of the traversed arcs,

\footnotetext{
${ }^{*}$ Research is supported by JST, ERATO, Kawarabayashi Large Graph Project, and by KAKENHI Grant Number 24106002, 24700004.

${ }^{\dagger}$ University of Tsukuba, Japan. E-mail: kobayashi@sk.tsukuba.ac.jp

${ }^{\ddagger}$ University of Tokyo, Japan. E-mail: sho_toyooka@mist.i.u-tokyo.ac.jp
} 
where each arc can be traversed in the reverse direction and then the label is inversed. Group labeled graphs are also called gain graphs or voltage graphs, and they were originally introduced in the field of topological graph theory with an application to construct graph embeddings in surfaces (see $[5,6,15]$ ). In this paper, we consider only abelian groups, and hence the group operation is denoted by addition and the identity is denoted by 0 . We now introduce the Shortest Non-Zero Path Problem, which is described as follows: given a group-labeled graph with two specified vertices $s$ and $t$, find an $s$ - $t$ path with a non-zero label that contains minimum number of arcs. This generalizes the shortest odd $s$ - $t$ path problem in undirected graphs with unit length edges, because odd $s$ - $t$ paths in an undirected graph $G$ correspond to non-zero $s$ - $t$ paths in the $\mathbb{Z}_{2}$-labeled graph obtained from $G$ by orienting each edge arbitrarily and by setting the label of each arc as 1. In this paper, we propose a polynomial time randomized algorithm for the Shortest Non-Zero Path Problem.

In order to state our result formally, we now give some notations. For an abelian group $\Gamma$, a $\Gamma$-labeled graph is a pair $(G, \psi)$ of a directed graph $G=(V, E)$ and a mapping $\psi: E \rightarrow \Gamma$ (called a label function). A walk in a $\Gamma$-labeled graph $(G, \psi)$ is a sequence $W=\left(v_{0}, e_{1}, v_{1}, e_{2}, v_{2}, \ldots, e_{l}, v_{l}\right)$ of vertices $v_{i}$ and $\operatorname{arcs} e_{i}$ in $G$ such that either $e_{i}=v_{i-1} v_{i}$ or $e_{i}=v_{i} v_{i-1}$ for $i=1, \ldots, l$. A path is a walk whose vertices are distinct from one another. The label of a walk $W=$ $\left(v_{0}, e_{1}, v_{1}, \ldots, e_{l}, v_{l}\right)$ is defined as $\psi(W)=\tilde{\psi}\left(e_{1}\right)+\tilde{\psi}\left(e_{2}\right)+\cdots+\tilde{\psi}\left(e_{l}\right)$, where $\tilde{\psi}\left(e_{i}\right)=\psi\left(e_{i}\right)$ if $e_{i}=v_{i-1} v_{i}$ and $\tilde{\psi}\left(e_{i}\right)=-\psi\left(e_{i}\right)$ if $e_{i}=v_{i} v_{i-1}$. The arc set of a walk $W$ is denoted by $E(W)$. With these notations, the Shortest Non-Zero Path Problem and our result are described as follows.

Shortest Non-Zero Path Problem in $\Gamma$-labeled Graphs

Input: a $\Gamma$-labeled graph $(G, \psi)$ with two specified vertices $s, t \in V$.

Find: an $s$ - $t$ path $P$ with $\psi(P) \neq 0$ that contains a minimum number of arcs (if exists).

Theorem 1. Let $\Gamma$ be a fixed finite abelian group. There is a polynomial time randomized algorithm for the Shortest Non-Zero Path Problem in $\Gamma$-labeled Graphs.

We note that $\Gamma$ is not a part of the input of the problem, that is, $|\Gamma|$ is a fixed constant when we consider polynomial solvability of the problem. We also note that we can easily find a shortest $s$ - $t$ walk with a non-zero label in polynomial time by standard dynamic programming. More precisely, for each integer $l$ and for each vertex $v \in V$, we compute all the possible labels of $s-v$ walks of length at most $l$. This can be done in polynomial time by using information on the case of $l-1$. Since this algorithm keeps no information on the intermediate vertices of $s-v$ walks, it is impossible to deal with paths instead of walks. Therefore, the same argument cannot be applied to the Shortest Non-Zero Path problem.

In our algorithm for the Shortest Non-Zero Path Problem, we introduce a matrix whose entries are polynomials, which is similar to the adjacency matrix. We reduce the Shortest NonZero Path problem to the computation of the permanent of this matrix modulo two under the assumption that the instance has a unique optimal solution (Proposition 4). This reduction is based on the ideas in the breakthrough paper by Björklund and Husfeldt [2], which gives a randomized polynomial time algorithm for the shortest two disjoint paths problem. Although computing the permanent of matrices is hard in general, it is shown in [2] that we can compute the permanent of one-variable polynomial matrices modulo two or four in polynomial time. Thus, we can solve the Shortest Non-Zero Path problem if the instance has a unique optimal solution. Even if the instance has more than one optimal solutions, we can convert it to an 
instance with a unique optimal solution with high probability by using a standard random perturbation technique and the isolation lemma (Lemma 6) in the same way as [2].

Since our algorithm is based on the permanent computation, we can say that our algorithm is algebraic rather than combinatorial. Linear algebraic objects such as determinant or permanent are closely related to some combinatorial optimization problems. One of the most classical examples is a relationship between the Tutte matrix and the maximum matching problem. It is well-known that the determinant of the Tutte matrix is non-zero if and only if the graph has a perfect matching [13], and we can obtain a randomized polynomial time algorithm for finding a perfect matching based on this relationship [10]. Later, this algorithm was derandomized in [4].

Permanent also appears in some combinatorial optimization problems. For example, the permanent of the adjacency matrix of a directed graph is equal to the number of cycle covers, and the permanent of the adjacency matrix of a bipartite graph is equal to the number of perfect matchings. However, since it is NP-hard to compute the permanent [14], these relationships do not lead to efficient algebraic algorithms. As far as we know, Björklund and Husfeldt's algorithm [2] mentioned above is the first efficient algorithm for a combinatorial optimization problem based on computing the permanent. Their key idea is to use the permanent modulo four, which can be computed in polynomial time. With such a background, the present paper aims to extend the applicability of the techniques in [2]. Indeed, we solve the Shortest NonZero Path Problem and the Shortest Non-Zero $k$ Disjoint Paths Problem, which are completely different from the problem in [2], by computing the permanent modulo $2^{r}$ for some integer $r$.

The rest of this paper is organized as follows. In Section 2, we give an algebraic algorithm for the Shortest Non-Zero Path Problem and prove Theorem 1. In Section 3, we extend our result to a kind of path packing problem, which we call the Shortest Non-Zero $k$ Disjoint Paths Problem. Note that this problem deals with $s$ - $t$ paths, whereas Björklund and Husfeldt [2] deal with two paths with distinct end vertices. In the algorithm for the Shortest Non-Zero $k$ Disjoint Paths Problem, we use an algorithm for computing the permanent of a polynomial matrix modulo $2^{r}$ for fixed integer $r$, which is given in Section 4 .

In what follows, by subdividing all arcs and assigning appropriate labels if necessary, we assume that the input graph contains neither self-loops nor parallel arcs without loss of generality.

\section{Algebraic Approach to the Problem}

In this section, we give a proof of Theorem 1, namely, we propose an algebraic approach to the Shortest Non-Zero Path Problem, in which we use the permanent of a polynomial matrix. The permanent of an $n \times n$ matrix $A=\left(a_{i j}\right)$ is defined as

$$
\text { per } A=\sum_{\sigma \in S_{n}} \prod_{i=1}^{n} a_{i \sigma(i)},
$$

where $S_{n}$ is the set of all permutations on $n$ elements. By the definition, the permanent of the adjacency matrix of a directed graph is corresponding to the number of cycle covers in this directed graph, where a cycle cover is a set of arcs in which each vertex has exactly one incoming arc and exactly one outgoing arc. More generally, we can easily see the following.

Lemma 2. Let $G=(V, E)$ be a directed graph which has no multiple arcs and may have parallel arcs and self-loops. Let $A=\left(a_{i j}\right)$ be a matrix whose rows and columns are indexed by $V$ such that $a_{i j}=0$ holds for any $i, j \in V$ with $i j \notin E$. Then, we have

$$
\text { per } A=\sum_{F \in \mathcal{C}(G)} \prod_{i j \in F} a_{i j},
$$


where $\mathcal{C}(G)$ is the set of all cycle covers in $G$.

To prove Theorem 1 , we first deal with the case of $\Gamma=\mathbb{Z}_{p}(:=\mathbb{Z} / p \mathbb{Z})$ for some integer $p$. We extend this case to the general case by using the fundamental theorem of finite abelian groups.

Suppose that we are given an instance of the Shortest Non-Zero Path Problem, that is, we are given a $\mathbb{Z}_{p}$-labeled graph $(G=(V, E), \psi)$ with two specified vertices $s, t \in V$. By identifying $\mathbb{Z}_{p}$ with $\{0,1,2, \ldots, p-1\} \subseteq \mathbb{Z}$, for each $i j \in E$, we regard $\psi(i j)$ as an integer with $0 \leq \psi(i j) \leq p-1$. We define a matrix $A=\left(a_{i j}\right)$ over $\mathbb{Z}[x, y]$ whose rows and columns are indexed by $V$ as follows:

$$
a_{i j}= \begin{cases}x y^{\psi(i j)} & \text { if } i j \in E, i \neq t, \text { and } j \neq s \\ x y^{p-\psi(j i)} & \text { if } j i \in E, i \neq t, \text { and } j \neq s \\ 1 & \text { if } i=j \in V \backslash\{s, t\} \\ 1 & \text { if }(i, j)=(t, s) \\ 0 & \text { otherwise. }\end{cases}
$$

Note that since $G$ has neither self-loops nor parallel arcs, $i j \in E$ implies that $i \neq j$ and $j i \notin E$, which ensures that $a_{i j}$ is well-defined. Since the maximum degree of $y$ in per $A$ is at most $|V| p$, per $A$ can be uniquely expressed as

$$
\text { per } A=\sum_{l=0}^{|V| p} q_{l}(x) y^{l},
$$

where $q_{l}(x)$ is a polynomial in $x$ with integer coefficients. With these polynomials, we define $Q(x)$ as the polynomial with coefficients in $\{0,1\}$ such that

$$
Q(x) \equiv \sum_{l \neq \equiv(\bmod p)} q_{l}(x) \quad(\bmod 2),
$$

where we denote $\sum_{i} b_{i} x^{i} \equiv \sum_{i} c_{i} x^{i}(\bmod 2)$ if $b_{i} \equiv c_{i}(\bmod 2)$ for every $i$.

Lemma 3. For a $\mathbb{Z}_{p}$-labeled graph $(G, \psi)$ with two vertices $s$ and $t, Q(x)$ defined above can be computed in polynomial time.

Proof. In order to compute $Q(x)$, we only need per $A$ modulo two, which can be computed as follows:

1. replace $y$ with $x^{N}$ to obtain a one-variable polynomial matrix $A^{\prime}$, where $N$ is greater than the maximum degree of $x$ in per $A$ (e.g., $N:=n+1$ ),

2. compute per $A^{\prime}$ modulo two, and

3. replace $x^{a N+b}$ with $x^{b} y^{a}$ in per $A^{\prime}$ to obtain per $A$ modulo two.

Since we can compute the permanent of one-variable polynomial matrices modulo two in polynomial time (see [2] or Section 4), this algorithm runs in polynomial time.

The following proposition shows a relationship between $Q(x)$ and the Shortest Non-Zero Path Problem.

Proposition 4. Suppose that we are given a $\mathbb{Z}_{p}$-labeled graph $(G, \psi)$ with two vertices $s$ and $t$, which is an instance of the Shortest Non-Zero Path Problem. Assume that it has a unique optimal solution. Then, the optimal value of this instance is equal to the minimum degree of $Q(x)$ defined as above. 
Proof. For an instance $(G=(V, E), \psi, s, t)$ of the Shortest Non-Zero Path Problem, we define a new directed graph $G^{\prime}=\left(V, E^{\prime}\right)$ with vertex set $V$ by

$$
\begin{aligned}
& E_{1}=\{i j \mid i j \in E, i \neq t, \text { and } j \neq s\}, \\
& E_{2}=\{i j \mid j i \in E, i \neq t, \text { and } j \neq s\}, \\
& E_{3}=\{i j \mid i=j \in V \backslash\{s, t\}\} \cup\{t s\}, \\
& E^{\prime}=E_{1} \cup E_{2} \cup E_{3} .
\end{aligned}
$$

We also define a new mapping $\psi^{\prime}: E^{\prime} \rightarrow\{0,1, \ldots, p-1\}$ by

$$
\psi^{\prime}(i j)= \begin{cases}\psi(i j) & \text { if } i j \in E_{1} \\ p-\psi(j i) & \text { if } i j \in E_{2} \\ 0 & \text { if } i j \in E_{3}\end{cases}
$$

for $i j \in E^{\prime}$. We can see that an $s$ - $t$ path $P$ in $\mathbb{Z}_{p}$-labeled graph $(G, \psi)$ is corresponding to a directed path $P^{\prime}$ from $s$ to $t$ (called an $s$ - $t$ dipath) in directed graph $G^{\prime}$ and their labels $\psi(P)$ and $\psi^{\prime}\left(P^{\prime}\right):=\sum_{e \in E\left(P^{\prime}\right)} \psi^{\prime}(e)$ are equal modulo $p$. Since $G^{\prime}$ and the matrix $A$ defined as (1) satisfy the condition in Lemma 2 , i.e., $i j \notin E^{\prime}$ implies that $a_{i j}=0$, we obtain

$$
\text { per } A=\sum_{F \in \mathcal{C}\left(G^{\prime}\right)} \prod_{i j \in F} a_{i j}
$$

where $\mathcal{C}\left(G^{\prime}\right)$ is the set of all cycle covers in $G^{\prime}$. We observe that a cycle cover $F \in \mathcal{C}\left(G^{\prime}\right)$ must contain the arc $t s$, and hence $F$ also contains an $s$ - $t$ dipath $P$. We now divide $\mathcal{C}\left(G^{\prime}\right)$ into two parts: one is the set $\mathcal{C}_{1}$ of all cycle covers containing an $s$ - $t$ dipath $P$ with $\psi^{\prime}(P) \neq 0$, and the other is the set $\mathcal{C}_{2}$ of all cycle covers containing an $s$ - $t$ dipath $P$ with $\psi^{\prime}(P)=0$. By (3), for each cycle cover $F \in \mathcal{C}\left(G^{\prime}\right)$, we can naturally define the contribution of $F$ to $Q(x)$, say $Q_{F}(x)$. That is, $Q_{F}(x)=0$ if $\sum_{e \in F} \psi^{\prime}(e) \equiv 0(\bmod p)$, and $Q_{F}(x)=x^{c_{F}}$ otherwise, where $c_{F}=\left|F \cap\left(E_{1} \cup E_{2}\right)\right|$. Then, we have $Q(x) \equiv \sum_{F \in \mathcal{C}\left(G^{\prime}\right)} Q_{F}(x)(\bmod 2)$ by the definition. In what follows, we consider $\sum_{F \in \mathcal{C}_{1}} Q_{F}(x)$ and $\sum_{F \in \mathcal{C}_{2}} Q_{F}(x)$, separately.

First, we consider $\sum_{F \in \mathcal{C}_{1}} Q_{F}(x)$. For an $s$-t dipath $P$ in $G^{\prime}$, let $A_{P}$ be the matrix obtained from $A$ by eliminating the rows and the columns corresponding to the vertices in $P$. Since each $F \in \mathcal{C}_{1}$ contains a non-zero $s$ - $t$ dipath, we have

$$
\begin{aligned}
\sum_{F \in \mathcal{C}_{1}} \prod_{i j \in F} a_{i j} & =\sum_{P: \text { non-zero s-t dipath }}\left(\prod_{i j \in E(P)} a_{i j}\right) \operatorname{per} A_{P} \\
& =\sum_{P: \text { non-zero } s-t \text { dipath }} x^{|E(P)|} y^{\bar{\psi}(P)} \operatorname{per} A_{P},
\end{aligned}
$$

where $\bar{\psi}(P)$ is some integer with $\bar{\psi}(P) \equiv \psi^{\prime}(P)(\bmod p)$. Consider the cycle cover $F_{0} \in \mathcal{C}_{1}$ that consists of the $s$ - $t$ dipath $P_{0}$ corresponding to the unique optimal solution (the shortest non-zero path) of the original problem, arc $t s$, and self-loops incident to vertices in $G^{\prime}-P_{0}$. Then, $Q_{F_{0}}(x)=x^{\left|E\left(P_{0}\right)\right|}$. By the uniqueness of the optimal solution and (4), we can see that $x^{\left|E\left(P_{0}\right)\right|}$ is the minimum degree term in $\sum_{F \in \mathcal{C}_{1}} Q_{F}(x)$ and its coefficient is 1.

Next, we show $\sum_{F \in \mathcal{C}_{2}} Q_{F}(x) \equiv 0(\bmod 2)$. Let $F \in \mathcal{C}_{2}$ be a cycle cover satisfying that $Q_{F}(x) \neq 0$. By the definition of $Q_{F}(x), \sum_{e \in F} \psi(e) \not \equiv 0(\bmod p)$ and $Q_{F}(x)=x^{c_{F}}$. Let $P$ be the $s$ - $t$ dipath with the label zero in $F$. We consider the cycle cover $F^{\prime} \in \mathcal{C}_{2}$ obtained from $F$ by reversing all arcs in $F-E(P)-t s$. Since $\sum_{e \in F^{\prime}} \psi(e) \equiv-\sum_{e \in F} \psi(e) \not \equiv 0(\bmod p)$, we have $Q_{F^{\prime}}(x)=x^{c_{F}}$, and hence $Q_{F}(x)+Q_{F^{\prime}}(x) \equiv 0(\bmod 2)$. Note that $F \neq F^{\prime}$, because 
$F-E(P)-t s$ contains at least one cycle whose label is not equal to zero. In this way, all cycle covers $F$ in $\mathcal{C}_{2}$ with $Q_{F}(x) \neq 0$ can be put into pairs so that the total contribution of each pair to $Q(x)$ is zero modulo two. Therefore, we obtain $\sum_{F \in \mathcal{C}_{2}} Q_{F}(x) \equiv 0(\bmod 2)$.

By the above analyses of $\sum_{F \in \mathcal{C}_{1}} Q_{F}(x)$ and $\sum_{F \in \mathcal{C}_{2}} Q_{F}(x)$, the minimum degree of $Q(x)$ is equal to the minimum length of the non-zero $s$ - $t$ path.

By combining Lemma 3 and Proposition 4, we obtain a deterministic polynomial time algorithm for the Shortest Non-Zero Path Problem under the assumption that the instance has a unique optimal solution. Even when a given instance has more than one optimal solutions, we can convert it to the case with a unique optimal solution by perturbing the lengths of the arcs.

Proposition 5. Suppose that we are given a $\Gamma$-labeled graph $(G, \psi)$ with two vertices $s$ and $t$, which is an instance of the Shortest Non-Zero Path Problem. We choose a weight $w(e)$ of each arc $e$ independently and uniformly at random from $W:=\{2|V||E|, 2|V||E|+1, \ldots, 2|V||E|+$ $2|E|-1\}$. We construct a new instance by replacing each arc e with a path of length $w(e)$, where the labels of the new arcs are chosen so that the label of the path is equal to $\psi(e)$. Then, the obtained instance has a unique optimal solution with probability at least $\frac{1}{2}$ (if the original instance has a feasible solution).

Proof. The validity of this proposition is based on the following isolation lemma [11]:

Lemma 6. Let $S$ be a finite set, $\mathcal{F}$ be a family of subsets of $S$, and $W$ be a set of integers different from each other. Suppose that the weight of each element in $S$ is chosen from $W$ independently and uniformly at random, then with probability at least $1-\frac{|S|}{|W|}$, there is a unique set in $\mathcal{F}$ of minimum total weight.

We apply this lemma, in which $S=E, W=\{2|V||E|, 2|V||E|+1, \ldots, 2|V||E|+2|E|-1\}$, and $\mathcal{F}$ is the family of all subsets of $E$ belonging to each $s$ - $t$ non-zero path in $G$. Then, with probability at least $1-\frac{|E|}{2|E|}=\frac{1}{2}$, there is a unique $s$ - $t$ non-zero path of minimum total weight in $G$. Since the total weight $\sum_{e \in E(P)} w(e)$ of an $s$ - $t$ path $P$ in $G$ is equal to the length of the corresponding path in the new instance, the obtained instance has a unique optimal solution with probability at least $\frac{1}{2}$.

Since an optimal solution in the instance obtained in Proposition 5 is corresponding to an optimal solution in the original instance, by Lemma 3 and Propositions 4 and 5, we obtain Theorem 1 under the assumption that $\Gamma=\mathbb{Z}_{p}$ for some integer $p$.

We now consider the case when $\Gamma$ is a finite abelian group. In this case, we apply the fundamental theorem of finite abelian groups and decompose $\Gamma$ as $\Gamma=\mathbb{Z}_{p_{1}} \oplus \mathbb{Z}_{p_{2}} \oplus \cdots \oplus \mathbb{Z}_{p_{l}}$ where $p_{1}, \ldots, p_{l}$ are some integers. Note that $l$ is bounded by a fixed constant since $\Gamma$ is fixed. By using this decomposition, for an instance $(G, \psi, s, t)$ of the Shortest Non-Zero Path Problem in $\Gamma$-labeled Graphs, define a new label function $\psi_{i}: E \rightarrow \mathbb{Z}_{p_{i}}$ for $i=1,2, \ldots, l$ such that $\psi(e)=\psi_{1}(e) \oplus \psi_{2}(e) \oplus \cdots \oplus \psi_{l}(e)$ for $e \in E$. For $i=1,2, \ldots, l$, let $P_{i}$ be a shortest nonzero $s$ - $t$ path in $\left(G, \psi_{i}\right)$. Then, a shortest one among $\left\{P_{1}, P_{2}, \ldots, P_{l}\right\}$ is a shortest non-zero $s$ - $t$ path in $(G, \psi)$, because a path $P$ satisfies that $\psi(P) \neq 0$ if and only if $\psi_{i}(P) \neq 0$ for some $i \in\{1,2, \ldots, l\}$. Therefore, by solving the Shortest Non-Zero Path Problem in $\mathbb{Z}_{p_{i}}$-labeled Graphs $\left(G, \psi_{i}\right)$ for $i=1,2, \ldots, l$, we obtain an optimal solution of the original problem in $(G, \psi)$, which shows Theorem 1 .

\section{$3 \quad$ Extension to Packing Disjoint $s$ - $t$ Paths}

In this section, we generalize the Shortest Non-Zero Path Problem to the problem of finding $k$ internally-disjoint $s$ - $t$ paths of shortest total length under the condition that the sum of their 
labels is not zero. We note that our result in this section does not imply the result in [2], because Björklund and Husfeldt [2] deal with the problem of finding an $s_{1}-t_{1}$ path and an $s_{2}-t_{2}$ path, which is different from our problem setting. Our problem is formally described as follows, where $k$ is a positive integer and $\Gamma$ is a finite abelian group.

\section{Shortest Non-Zero $k$ Disjoint Paths Problem in $\Gamma$-labeled Graphs}

Input: a $\Gamma$-labeled graph $(G, \psi)$ with two specified vertices $s, t \in V$.

Find: $k$ internally-disjoint $s$ - $t$ paths $P_{1}, \ldots, P_{k}$ minimizing the total number of arcs contained in them subject to $\sum_{i=1}^{k} \psi\left(P_{i}\right) \neq 0$ (if exist).

We can easily see that the case of $k=1$ is corresponding to the Shortest Non-Zero Path Problem. The objective of this section is to extend Theorem 1 to the following theorem.

Theorem 7. Let $k$ be a fixed positive integer and $\Gamma$ be a fixed finite abelian group. There is a polynomial time randomized algorithm for the Shortest Non-Zero $k$ Disjoint Paths Problem in T-labeled Graphs.

Proof. By subdividing all arcs and assigning appropriate labels if necessary, we may assume that the input graph contains neither self-loops nor parallel arcs and there is no arc connecting $s$ and $t$ without loss of generality. By using the same argument as the previous section, it suffices to discuss the case of $\Gamma=\mathbb{Z}_{p}$. Suppose that we are given an instance of the Shortest Non-Zero $k$ Disjoint Paths Problem. We construct a new graph $G^{\prime}=\left(V^{\prime}, E^{\prime}\right)$ from $G$ by replacing $s$ with its $k$ copies $s_{1}, s_{2}, \ldots, s_{k}$ and by replacing $t$ with its $k$ copies $t_{1}, t_{2}, \ldots, t_{k}$. Note that each arc incident to $s$ (resp. $t$ ) is also replaced with its $k$ copies incident to $s_{i}$ (resp. $t_{i}$ ), and the label function $\psi$ on $E$ is naturally extended to $E^{\prime}$ (see Figure 1). Define $S=\left\{s_{1}, s_{2}, \ldots, s_{k}\right\}$ and $T=\left\{t_{1}, t_{2}, \ldots, t_{k}\right\}$.

Recall that, for each $i j \in E^{\prime}$, we can regard $\psi(i j)$ as an integer with $0 \leq \psi(i j) \leq p-1$. We define a matrix $A^{\prime}=\left(a_{i j}^{\prime}\right)$ over $\mathbb{Z}[x, y]$ whose rows and columns are indexed by $V^{\prime}$ as follows:

$$
a_{i j}^{\prime}= \begin{cases}x y^{\psi(i j)} & \text { if } i j \in E^{\prime}, i \notin T, \text { and } j \notin S \\ x y^{p-\psi(j i)} & \text { if } j i \in E^{\prime}, i \notin T, \text { and } j \notin S \\ 1 & \text { if } i=j \in V^{\prime} \backslash(S \cup T) \\ 1 & \text { if }(i, j)=\left(t_{l}, s_{l}\right) \text { for some } l \in\{1,2, \ldots, k\} \\ 0 & \text { otherwise. }\end{cases}
$$

In a similar way to $(2)$, we express per $A^{\prime}$ as

$$
\operatorname{per} A^{\prime}=\sum_{l=0}^{\left|V^{\prime}\right| p} q_{l}^{\prime}(x) y^{l}
$$

and define $Q^{\prime}(x)$ as the polynomial with coefficients in $\left\{0,1,2, \ldots, 2^{r}-1\right\}$ such that

$$
Q^{\prime}(x) \equiv \sum_{l \neq 0(\bmod p)} q_{l}^{\prime}(x) \quad\left(\bmod 2^{r}\right) .
$$

Here, $r$ is the minimum integer such that $(k !)^{2} / 2^{r}$ is not an integer, i.e., $(k !)^{2} \not \equiv 0\left(\bmod 2^{r}\right)$ and $2(k !)^{2} \equiv 0\left(\bmod 2^{r}\right)$. In a similar way to Proposition 4 , we can obtain the following proposition. 

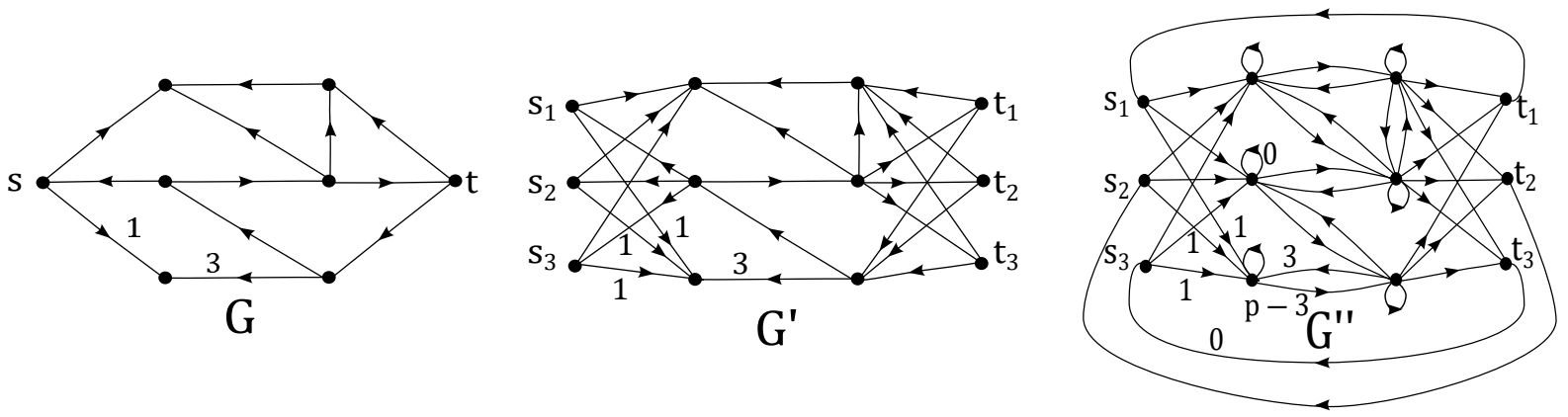

Figure 1: Construction of $G^{\prime}$ and $G^{\prime \prime}$

Proposition 8. Let $k$ be a positive integer. Suppose that we are given a $\mathbb{Z}_{p}$-labeled graph $G=(V, E)$ with two vertices $s$ and $t$, which is an instance of the Shortest Non-Zero $k$ Disjoint Paths Problem. Assume that it has a unique optimal solution. Then, the optimal value of this instance is equal to the minimum degree of $Q^{\prime}(x)$ defined as above.

Proof of Proposition 8. For the group-labeled graph $\left(G^{\prime}, \psi\right)$ defined as above, as in Figure 1, we define a new directed graph $G^{\prime \prime}=\left(V^{\prime}, E^{\prime \prime}\right)$ with vertex set $V^{\prime}$ by

$$
\begin{aligned}
& E_{1}=\left\{i j \mid \text { if } i j \in E^{\prime}, i \notin T, \text { and } j \notin S\right\}, \\
& E_{2}=\left\{i j \mid \text { if } j i \in E^{\prime}, i \notin T, \text { and } j \notin S\right\}, \\
& \left.E_{3}=\left\{i j \mid i=j \in V^{\prime} \backslash(S \cup T)\right\}\right\} \cup\left\{t_{l} s_{l} \mid l \in\{1,2, \ldots, k\}\right\}, \\
& E^{\prime \prime}=E_{1} \cup E_{2} \cup E_{3} .
\end{aligned}
$$

We also define a new mapping $\psi^{\prime \prime}: E^{\prime \prime} \rightarrow\{0,1, \ldots, p-1\}$ by

$$
\psi^{\prime \prime}(i j)= \begin{cases}\psi(i j) & \text { if } i j \in E_{1} ; \\ p-\psi(j i) & \text { if } i j \in E_{2} ; \\ 0 & \text { if } i j \in E_{3}\end{cases}
$$

for $i j \in E^{\prime \prime}$. Since $G^{\prime \prime}$ and the matrix $A^{\prime}$ defined as (5) satisfy the condition in Lemma 2, i.e., $i j \notin E^{\prime \prime}$ implies that $a_{i j}^{\prime}=0$, we obtain

$$
\operatorname{per} A^{\prime}=\sum_{F \in \mathcal{C}\left(G^{\prime \prime}\right)} \prod_{i j \in F} a_{i j}^{\prime},
$$

where $\mathcal{C}\left(G^{\prime \prime}\right)$ is the set of all cycle covers in $G^{\prime \prime}$. We observe that a cycle cover $F \in \mathcal{C}\left(G^{\prime \prime}\right)$ must contain the arc $t_{l} s_{l}$ for $l=1,2, \ldots, k$, and hence $F$ also contains $k$ (fully) disjoint directed paths from $S$ to $T$, which we call $S-T$ dipaths. We now divide $\mathcal{C}\left(G^{\prime \prime}\right)$ into two parts: one is the set $\mathcal{C}_{1}^{\prime}$ of all cycle covers containing $S$ - $T$ dipaths whose sum of the labels is non-zero (non-zero $S-T$ dipaths), and the other is the set $\mathcal{C}_{2}^{\prime}$ of all cycle covers containing $S-T$ dipaths whose sum of the labels is zero (zero $S-T$ dipaths). In the same way as the proof of Proposition 4, for each cycle cover $F \in \mathcal{C}\left(G^{\prime \prime}\right)$, we can naturally define the contribution of $F$ to $Q^{\prime}(x)$, say $Q_{F}^{\prime}(x)$. In what follows, we consider $\sum_{F \in \mathcal{C}_{1}^{\prime}} Q_{F}^{\prime}(x)$ and $\sum_{F \in \mathcal{C}_{2}^{\prime}} Q_{F}^{\prime}(x)$, separately.

First, we consider $\sum_{F \in \mathcal{C}_{1}^{\prime}} Q_{F}^{\prime}(x)$. For a set $\mathcal{P}$ of dipaths in $G^{\prime \prime}$, let $E(\mathcal{P})$ be the set of the arcs contained in the dipaths in $\mathcal{P}$, define $\psi^{\prime \prime}(\mathcal{P}):=\sum_{e \in E(\mathcal{P})} \psi^{\prime \prime}(e)$, and let $A_{\mathcal{P}}^{\prime}$ be the matrix obtained from $A^{\prime}$ by eliminating the rows and the columns corresponding to the vertices in the dipaths in $\mathcal{P}$. Since each $F \in \mathcal{C}_{1}^{\prime}$ contains non-zero $S$ - $T$ dipaths, we have

$$
\sum_{F \in \mathcal{C}_{1}^{\prime}} \prod_{i j \in F} a_{i j}^{\prime}=\sum_{\mathcal{P}: \text { non-zero } S-T \text { dipaths }} x^{|E(\mathcal{P})|} y^{\bar{\psi}(\mathcal{P})} \text { per } A_{\mathcal{P}}^{\prime}
$$


where $\bar{\psi}(\mathcal{P})$ is some integer with $\bar{\psi}(\mathcal{P}) \equiv \psi^{\prime \prime}(\mathcal{P})(\bmod p)$. Let $\mathcal{P}_{0}$ be the unique optimal solution of the Shortest Non-Zero $k$ Disjoint Paths Problem. Consider a cycle cover $F_{0} \in \mathcal{C}_{1}^{\prime}$ in $G^{\prime \prime}$ that consists of non-zero $S$-T paths $\mathcal{P}$ corresponding to $\mathcal{P}_{0}$, arcs $t_{l} s_{l}(l=1, \ldots, k)$, and selfloops incident to vertices not contained in $\mathcal{P}$. Then, $Q_{F_{0}}^{\prime}(x)=x^{\left|E\left(\mathcal{P}_{0}\right)\right|}$. Since we can obtain $\mathcal{P}$ from $\mathcal{P}_{0}$ by determining a one-to-one correspondence between $\mathcal{P}_{0}$ and $S$ and a one-to-one correspondence between $\mathcal{P}_{0}$ and $T$, we have $(k !)^{2}$ possibilities of $F_{0}$ with the above condition. Therefore, $(k !)^{2} x^{\left|E\left(\mathcal{P}_{0}\right)\right|}$ is the minimum degree term in $\sum_{F \in \mathcal{C}_{1}^{\prime}} Q_{F}^{\prime}(x)$. Note that $(k !)^{2} \not \equiv 0$ $\left(\bmod 2^{r}\right)$ by the definition of $r$.

Next, we show $\sum_{F \in \mathcal{C}_{2}^{\prime}} Q_{F}^{\prime}(x) \equiv 0\left(\bmod 2^{r}\right)$. Let $F \in \mathcal{C}_{2}^{\prime}$ be a cycle cover satisfying that $Q_{F}^{\prime}(x) \neq 0$. Then, $\sum_{e \in F} \psi^{\prime \prime}(e) \not \equiv 0(\bmod p)$ and $Q_{F}^{\prime}(x)=x^{c_{F}}$, where $c_{F}=\left|F \cap\left(E_{1} \cup E_{2}\right)\right|$. By changing the indices of $\left\{s_{1}, \ldots, s_{k}\right\}$ and $\left\{t_{1}, \ldots, t_{k}\right\}$ in $F$, we obtain $(k !)^{2}$ cycle covers $F_{1}(=F), F_{2}, \ldots, F_{(k !)^{2}} \in \mathcal{C}_{2}^{\prime}$ such that $Q_{F_{i}}^{\prime}(x)=x^{c_{F}}$ for $i=1,2, \ldots,(k !)^{2}$. Note that these cycle covers are distinct since the original graph has no arc connecting $s$ and $t$. Let $\mathcal{P}$ be the zero $S$ - $T$ dipaths in $F$, and consider the cycle cover $F^{\prime} \in \mathcal{C}_{2}^{\prime}$ obtained from $F$ by reversing all $\operatorname{arcs}$ in $F-E(\mathcal{P})-\left\{t_{1} s_{1}, \ldots, t_{k} s_{k}\right\}$. Since $\sum_{e \in F^{\prime}} \psi^{\prime \prime}(e) \equiv-\sum_{e \in F} \psi^{\prime \prime}(e) \not \equiv 0(\bmod p)$, we have $Q_{F^{\prime}}(x)=x^{c_{F}}$. Again, by changing the indices of $\left\{s_{1}, \ldots, s_{k}\right\}$ and $\left\{t_{1}, \ldots, t_{k}\right\}$ in $F^{\prime}$, we have $(k !)^{2}$ cycle covers $F_{1}^{\prime}\left(=F^{\prime}\right), F_{2}^{\prime}, \ldots, F_{(k !)^{2}}^{\prime} \in \mathcal{C}_{2}^{\prime}$ such that $Q_{F_{i}^{\prime}}^{\prime}(x)=x^{c_{F}}$ for $i=1,2, \ldots,(k !)^{2}$. Therefore, $\sum_{i=1}^{(k !)^{2}}\left(Q_{F_{i}}(x)+Q_{F_{i}^{\prime}}(x)\right)=2(k !)^{2} x^{c_{F}} \equiv 0\left(\bmod 2^{r}\right)$, since $2(k !)^{2} \equiv 0\left(\bmod 2^{r}\right)$ by the definition of $r$. In this way, all cycle covers $F$ in $\mathcal{C}_{2}^{\prime}$ with $Q_{F}^{\prime}(x) \neq 0$ can be divided into sets of $2(k !)^{2}$ cycle covers so that the total contribution of each set to $Q^{\prime}(x)$ is zero modulo $2^{r}$. Therefore, we obtain $\sum_{F \in \mathcal{C}_{2}^{\prime}} Q_{F}^{\prime}(x) \equiv 0\left(\bmod 2^{r}\right)$.

By the above analyses of $\sum_{F \in \mathcal{C}_{1}^{\prime}} Q_{F}^{\prime}(x)$ and $\sum_{F \in \mathcal{C}_{2}^{\prime}} Q_{F}^{\prime}(x)$, the minimum degree of $Q^{\prime}(x)$ is equal to the optimal value of the Shortest Non-Zero $k$ Disjoint Paths Problem. (End of the proof of Proposition 8)

In order to compute $Q^{\prime}(x)$ modulo $2^{r}$, in a similar way to Lemma 3 , we convert $A^{\prime}$ to a matrix in $\mathbb{Z}[x]$ and compute its permanent modulo $2^{r}$. This can be done in polynomial time as we will see in the next section (see Theorem 9). Note that the degree of each element of the obtained matrix is at most $O\left(\left|V^{\prime}\right|^{2}\right)$, and hence the degree of the permanent is at most $O\left(\left|V^{\prime}\right|^{3}\right)$, which is needed when we apply Theorem 9. Therefore, by Proposition 8 and the perturbation technique used in Section 2, we obtain Theorem 7.

\section{Computing the Permanent Modulo $2^{r}$}

For the computation of $Q^{\prime}(x)$, we propose an algorithm for computing the permanent of polynomial matrices modulo $2^{r}$, which we believe is of independent interest.

Although computing the permanent of integer matrices is NP-hard [14], Valiant [14] gave a polynomial time algorithm for computing the permanent of matrices whose entries are in $\mathbb{Z}_{2^{r}}$, where $r$ is a fixed constant. By using a similar technique to [14], Björklund and Husfeldt [2] gave a polynomial time algorithm for computing the permanent of matrices whose entries are in $\mathbb{Z}_{4}[x]$, that is, each entry is a polynomial in $x$ with coefficients in $\mathbb{Z}_{4}$. Our contribution is to generalize this result to the case of $\mathbb{Z}_{2^{r}}[x]$, where $r$ is a fixed constant. For a matrix $A$ whose entries are in $\mathbb{Z}[x]$ and for a positive integer $r$, let $\operatorname{per}_{2^{r}} A$ be the permanent of $A$ modulo $2^{r}$, i.e., the polynomial with coefficients in $\left\{0,1,2, \ldots, 2^{r}-1\right\}$ such that

$$
\operatorname{per}_{2^{r}} A \equiv \operatorname{per} A \quad\left(\bmod 2^{r}\right) .
$$

Our result is stated as follows. 
Theorem 9. Let $r$ be a fixed nonnegative integer and $A$ be an $n \times n$ matrix whose entries are in $\mathbb{Z}[x]$. Suppose that we are given an integer $N$ which is greater than the maximum degree of $\operatorname{per}_{2^{r}} A$. Then, $\operatorname{per}_{2^{r}} A$ can be computed in polynomial time in $n$ and $N$.

Proof. Our proof is based on ideas in [2]. Let $E_{N}$ denote $\mathbb{Z}[x] /\left(x^{N}\right)$, which is a quotient ring divided by the ideal generated by $x^{N}$. Roughly, $E_{N}$ is the set of polynomials obtained from $\mathbb{Z}[x]$ by ignoring the terms whose degrees are at least $N$. Since the maximum degree of $\operatorname{per}_{2^{r}} A$ is at most $N-1$, to compute $\operatorname{per}_{2^{r}} A$, we may identify $\mathbb{Z}[x]$ with $E_{N}$ by ignoring the terms whose degrees are at least $N$. Let $\mathrm{M}_{n}\left(E_{N}\right)$ be the set of all $n \times n$ matrices whose entries are in $E_{N}$. We say that a polynomial $a \in E_{N}$ is even if all coefficients of $a$ are even and odd if $a$ is not even. For an odd polynomial $a$, let $m(a)$ be the index of the lowest order term of $a$ whose coefficient is odd.

For a given matrix $A=\left(a_{i j}\right) \in \mathrm{M}_{n}\left(E_{N}\right)$, our algorithm for computing $\operatorname{per}_{2^{r}} A$ is $\operatorname{described}$ as follows. Note that all the computation in the algorithm is done on $E_{N}$, that is, we remove all terms whose degrees are at least $N$.

Algorithm Permanent $(r, A)$

A1. If $n=1$, return $a_{11}$ modulo $2^{r}$. If $r=0$, return 0 .

A2. Choose $i \in\{1,2, \ldots, n\}$ such that $a_{i 1}$ is odd and $m\left(a_{i 1}\right)$ is minimum (if exists). Then, exchange rows 1 and $i$.

A3. If $a_{i 1}$ is even for $i=2,3 \ldots, n$, then compute $\operatorname{per}_{2^{r}} A$ by Lemma 10 and return it. Otherwise, take an index $i \in\{2,3, \ldots, n\}$ such that $a_{i 1}$ is odd, and compute a polynomial $c \in E_{N}$ such that $a_{i 1}+c a_{11} \in E_{N}$ is even by Lemma 11 .

A4. Let $A[i, 1]$ be the matrix obtained from $A$ by replacing the $i$ th row with the first row.

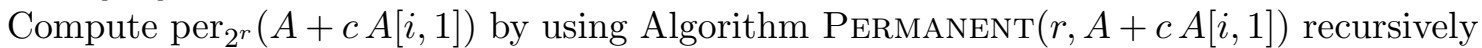
and compute $c \operatorname{per}_{2^{r}} A[i, 1]$ by Lemma 12 . Then, compute $\operatorname{per}_{2^{r}} A$ by

$$
\operatorname{per}_{2^{r}} A \equiv \operatorname{per}_{2^{r}}(A+c A[i, 1])-c \operatorname{per}_{2^{r}} A[i, 1] \quad\left(\bmod 2^{r}\right),
$$

and return it.

For integers $n \geq 1, r \geq 0$, and $k \geq 0$, let $T_{N}(n, r, k)$ be the worst case running time of the algorithm for computing $\operatorname{per}_{2^{r}} A$ for a matrix $A=\left(a_{i j}\right) \in \mathrm{M}_{n}\left(E_{N}\right)$ such that $\mid\{i \in\{1,2, \ldots n\} \mid$ $a_{i 1}$ is odd $\} \mid$ is at most $k$. Note that $T_{N}$ is monotone, that is, $T_{N}(n, r, k) \geq T_{N}\left(n^{\prime}, r^{\prime}, k^{\prime}\right)$ if $n \geq n^{\prime}$, $r \geq r^{\prime}$, and $k \geq k^{\prime}$. For each $n$ and each $r$, let $T_{N}^{*}(n, r):=\max _{k} T_{N}(n, r, k)\left(=T_{N}(n, r, n)\right)$. In what follows, we prove that $T_{N}^{*}(n, r)$ is bounded by a polynomial in $n$ and $N$ for fixed $r$. Let poly $(n, N)$ denote some polynomial in $n$ and $N$. Note that when poly $(n, N)$ appears more than once, they might denote distinct polynomials.

The following lemmas are used in Algorithm $\operatorname{Permanent}(r, A)$.

Lemma 10. Let $n \geq 2$ and $r \geq 1$ be integers and $A=\left(a_{i j}\right)$ be a matrix in $\mathrm{M}_{n}\left(E_{N}\right)$. If $a_{i 1}$ is even for $i=2,3 \ldots, n$, then we can compute $\operatorname{per}_{2^{r}} A$ in $T_{N}^{*}(n-1, r)+(n-1) T_{N}^{*}(n-1, r-1)+\operatorname{poly}(n, N)$ time. That is, $T_{N}(n, r, 1) \leq T_{N}^{*}(n-1, r)+(n-1) T_{N}^{*}(n-1, r-1)+\operatorname{poly}(n, N)$ for $n \geq 2$ and $r \geq 1$.

Proof. By expanding per $A$ along the first column, we have

$$
\operatorname{per}_{2^{r}} A \equiv a_{11} \operatorname{per}_{2^{r}} A_{11}+\sum_{i=2}^{n} a_{i 1} \operatorname{per}_{2^{r}} A_{i 1} \quad\left(\bmod 2^{r}\right),
$$


where $A_{i 1}$ is the matrix obtained from $A$ by removing row $i$ and column 1 . For $i=2,3, \ldots, n$, since $a_{i 1}$ is even, we have

$$
a_{i 1} \operatorname{per}_{2^{r}} A_{i 1} \equiv a_{i 1} \operatorname{per}_{2^{r-1}} A_{i 1} \quad\left(\bmod 2^{r}\right) .
$$

This shows that we can compute $(7)$ in $T_{N}^{*}(n-1, r)+(n-1) T_{N}^{*}(n-1, r-1)+\operatorname{poly}(n, N)$ time.

Lemma 11. For odd polynomials $a \in E_{N}$ and $b \in E_{N}$ with $m(a) \leq m(b)$, we can compute $a$ polynomial $c \in E_{N}$ such that $b+c a \in E_{N}$ is even in polynomial time in $N$.

Proof. Such a $c$ can be computed by the following algorithm.

B1. Set $l=0$ and $c^{(0)}=0 \in E_{N}$.

B2. While $b+c^{(l)} a$ is not even, set $c^{(l+1)}=c^{(l)}+x^{m\left(b+c^{(l)} a\right)-m(a)}$ and increment $l$.

B3. Return $c^{(l)}$.

Since each iteration in Step B2 increases $m\left(b+c^{(l)} a\right)$ by at least one and this value is at most $N-1$, this algorithm runs in polynomial time in $N$.

Lemma 12. Let $n \geq 2$ and $r \geq 1$ be integers and $A=\left(a_{i j}\right)$ be a matrix in $\mathrm{M}_{n}\left(E_{N}\right)$ whose first and second rows are identical. Then,

$$
\operatorname{per}_{2^{r}} A \equiv 2 \sum_{1 \leq i<j \leq n} a_{1 i} a_{2 j} \operatorname{per}_{2^{r-1}} A_{1 i, 2 j} \quad\left(\bmod 2^{r}\right),
$$

where $A_{1 i, 2 j}$ is the matrix obtained from $A$ by removing rows 1 and 2 and columns $i$ and $j$. Furthermore, $\operatorname{per}_{2^{r}} A$ can be computed in $\frac{1}{2} n(n-1) T_{N}^{*}(n-2, r-1)+\operatorname{poly}(n, N)$ time, where $T_{N}^{*}(0, r-1)$ is regarded as a constant.

Proof of Lemma 12. By expanding per $A$ along the first and second rows,

$$
\begin{aligned}
\operatorname{per}_{2^{r}} A & \equiv \sum_{i \neq j} a_{1 i} a_{2 j} \operatorname{per}_{2^{r}} A_{1 i, 2 j} \\
& \equiv 2 \sum_{1 \leq i<j \leq n} a_{1 i} a_{2 j} \operatorname{per}_{2^{r}} A_{1 i, 2 j} \\
& \equiv 2 \sum_{1 \leq i<j \leq n} a_{1 i} a_{2 j} \operatorname{per}_{2^{r-1}} A_{1 i, 2 j} \quad\left(\bmod 2^{r}\right),
\end{aligned}
$$

where the last equality is derived from the fact that $2 a \equiv 2 a^{\prime}\left(\bmod 2^{r}\right)$ if and only if $a \equiv a^{\prime}$ $\left(\bmod 2^{r-1}\right)$ for $a, a^{\prime} \in E_{N}$. Since $\operatorname{per}_{2^{r-1}} A_{1 i, 2 j}$ can be computed in $T_{N}^{*}(n-2, r-1)$ time, $\operatorname{per}_{2^{r}} A$ can be computed in $\frac{1}{2} n(n-1) T_{N}^{*}(n-2, r-1)+\operatorname{poly}(n, N)$ time. (End of the proof of Lemma 12)

Now we are ready to evaluate $T_{N}(n, r, k)$ and prove Theorem 9 . For $k \geq 2$ and $r \geq 1$, by Step A4 of Algorithm Permanent $(r, A)$ and Lemma 12, we obtain

$$
T_{N}(n, r, k) \leq T_{N}(n, r, k-1)+\frac{1}{2} n(n-1) T_{N}^{*}(n-2, r-1)+\operatorname{poly}(n, N) .
$$


By using this inequality repeatedly, it holds that

$$
\begin{aligned}
T_{N}(n, r, k) & \leq T_{N}(n, r, 1)+\frac{k-1}{2} n(n-1) T_{N}^{*}(n-2, r-1)+\operatorname{poly}(n, N) \\
& \leq T_{N}(n, r, 1)+\frac{n^{3}}{2} T_{N}^{*}(n-2, r-1)+\operatorname{poly}(n, N) .
\end{aligned}
$$

Note that this inequality holds also for $k=0,1$. By combining (8) with Lemma 10, we have

$$
\begin{aligned}
T_{N}(n, r, k) & \leq T_{N}^{*}(n-1, r)+(n-1) T_{N}^{*}(n-1, r-1)+\frac{n^{3}}{2} T_{N}^{*}(n-2, r-1)+\operatorname{poly}(n, N) \\
& \leq T_{N}^{*}(n-1, r)+n^{3} T_{N}^{*}(n, r-1)+\operatorname{poly}(n, N),
\end{aligned}
$$

where we use the monotonicity of $T_{N}^{*}$ in the second inequality. Since this inequality holds for any $k \geq 0$, we have

$$
T_{N}^{*}(n, r) \leq T_{N}^{*}(n-1, r)+n^{3} T_{N}^{*}(n, r-1)+\operatorname{poly}(n, N)
$$

for any $n$ and $r$. By using (9) repeatedly (by changing $n$ ), we obtain

$$
T_{N}^{*}(n, r) \leq n^{4} T_{N}^{*}(n, r-1)+\operatorname{poly}(n, N) .
$$

Furthermore, by using (10) repeatedly (by changing $r$ ), we obtain $T_{N}^{*}(n, r)=(\operatorname{poly}(n, N))^{O(r)}$. This shows that Algorithm Permanent $(r, A)$ runs in polynomial time in $n$ and $N$ for fixed $r$.

\section{References}

[1] R.E. Bellman, On a routing problem, Quarterly of Applied Mathematics (1958) 16, pp. $87-90$.

[2] A. Buörklund And T. Husfeldt, Shortest two disjoint paths in polynomial time, Proceedings of the 41st International Colloquium on Automata, Languages and Programming, Part I. LNCS 8572 (2014), pp. 211-222.

[3] E.W. Dijkstra, A note on two problems in connexion with graphs, Numerische Mathematik (1959) 1, pp. 269-271.

[4] J.F. Geelen, An algebraic matching algorithm, Combinatorica (2000) 20, pp. 61-70.

[5] J.L Gross And T.W. Tucker, Generating all graph coverings by permutation voltage assignments, Discrete Mathematics (1977) 18, pp. 273-283.

[6] J.L Gross and T.W. Tucker, Topological Graph Theory, Wiley Interscience, 1987.

[7] M. Grötschel and W.R. Pulleyblank, Weakly bipartite graphs and the max-cut problem, Operations Research Letters (1981) 1, pp. 23-27.

[8] T. Huynh, The Linkage Problem for Group-Labelled Graphs PhD. Thesis, Department of Combinatorics and Optimization, University of Waterloo, Ontario, 2009.

[9] A.S. LaPaugh and C.H. Papadimitriou, The even-path problem for graphs and digraphs, Networks (1984) 14, pp. 507-513. 
[10] L. LovÁsz, On determinants, matchings, and random algorithms, Fundamentals of Computation Theory, FCT 'r9, Akademie-Verlag, Berlin, 1979, pp. 565-574.

[11] K. Mulmuley, U.V. Vazirani, and V.V. Vazirani, Matching is as easy as matrix inversion, Combinatorica (1987) 7, pp. 105-113.

[12] A. SchriJver, Combinatorial Optimization. Polyhedra and Efficiency, Springer-Verlag, 2003.

[13] W.T. TutTe, The factorization of linear graphs, Journal of the London Mathematical Society (1947) 22, pp. 107-111.

[14] L.G. Valiant, The complexity of computing the permanent, Theoretical Computer Science (1979) 8, pp. 189-201.

[15] T. Zaslavsky, Biased graph. I. bias, balance, and gains, Journal of Combinatorial Theory, Series B (1989) 47, pp. 32-52. 